\title{
A STUDY COMPARING THE EFFECTIVENESS OF THE FELDENKRAIS METHOD VERSUS THE STANDARD PULMONARY REHABILITATION PROGRAM IN IMPROVING THE BORG SCORE AND 6 MINUTE WALK IN PATIENTS WITH CHRONIC OBSTRUCTIVE PULMONARY DISEASE (COPD)
}

\author{
Ayiesah $R^{1}$, Roslina $A M^{2}$ \\ 1 Physiotherapy Programme, Faculty of Allied Health Sciences, Universiti Kebangsaan Malaysia, Kuala Lumpur \\ 2 Respiratory Department, Hospital Universiti Kebangsaan Malaysia, UKM, Cheras, Selangor
}

\section{Correspondence:}

Associate Professor Dr (Hjh) Ayiesah Hj. Ramli

Physiotherapy Programme

Faculty of Health Sciences

Universiti Kebangsaan Malaysia

Jalan Raja Muda Abdul Aziz

50300 Kuala Lumpur, Malaysia

Email: kamalia086@gmail.com

Tel: 603-2687 8003; 016-535 1002

Fax: 603-2687 7819

\begin{abstract}
INTRODUCTION:

Although the Feldenkrais method of rehabilitating chronic obstructive pulmonary disease (COPD) patients have been suggested, its use among practitioners is not widespread owing to preference of the more familiar standard program presently available. Several advantageous of the Feldenkrais Method have been suggested which includes improving the efficiency of movement, posture and, breathing. However how this compares to the standard rehabilitation protocol or pulmonary rehabilitation program (PRP) have not been previously demonstrated. The present study was thus conducted to compare the effectiveness of the Feldenkrais Method to the standard PRP using Borg score and 6 minute walked distance (6MWD) as outcome measurement tools.
\end{abstract}

\section{RESULTS:}

There were 17 subjects in the Feldenkrais group (FG) and 19 subjects in the pulmonary group (PG), both of which received therapy for 8 weeks and assessed before and after receiving therapy. There were no improvements observed in the Borg score for the FG (after and before; $6.06 \pm 1.09$ vs. $6.00 \pm 0.94$ ). However, improvements in the PG group could be seen (after and before; $3.58 \pm 1.17$ vs. $5.84 \pm 1.01$ ). Improvements in the 6MWD was observed in both groups with no significance differences noted (FG vs. PG; $379 \pm 129 \mathrm{~m}$ vs. $374 \pm 80 \mathrm{~m}$ ).

\section{CONCLUSION:}

The Feldenkrais method does not offer any advantage over the present PRP and in fact the latter offers better improvement in terms of the Borg score. However in view of the small study sample, further study would be needed before a final conclusion can be made.

Keywords: Borg scale, exercise tolerance, Feldenkrais method, 6 minute walk test, pulmonary rehabilitation program 


\section{Introduction}

Pulmonary rehabilitation program (PRP) for chronic obstructive pulmonary disease (COPD) patients is well established $(1,2)$. It is a widely accepted therapeutic means of enhancing standard therapy to lessen symptoms and optimize function. Such programs have encouraged patients to become more independent in their daily activities, and become less dependent on health professionals and expensive medical resources (2). The problems associated with COPD such as exercise deconditioning, muscle wasting, dyspnoea and weight loss can be overcomed with the attendance to PRPs (3).

The Feldenkrais Method is becoming an increasingly popular treatment modality used by health professionals to assess a range of dysfunctions (4). It was originally developed to explore the relationship between movement, physical experience, and development. This modality helps to decrease the muscular effort that is needed to perform movement and is directed at those people who wish to improve their efficiency of movement, posture and breathing, muscular tension, flexibility and neuromotor functioning (5).

Dyspnoea has been the primary reason for COPD patients seeking medical care. Measurements of dyspnoea provide an insight into the practical effects of treatment on everyday life, reflecting whether or not the patients perceive an improvement in their primary symptom. Mostly, the COPD patients would often decrease their activity to avoid the unpleasant sensation of breathlessness. Several methods have been used to evaluate patient's experience of breathlessness, during daily activities (using of questionnaires) or exercise testing (the Borg scale)(6). These measures have been produced to grade the severity of dyspnoea according to the degree of breathlessness associated with any particular task.

In clinical practice, the 6-minute walk test (6MWT) is commonly used to assess changes in functional exercise capacity following pulmonary rehabilitation. Both tests (6MWT and Borg score) have showed validity, reliability after one familiarization test, and have the ability to detect changes following pulmonary rehabilitation (7). Unlike pulmonary rehabilitation, there is a relatively limited body of empirical evidence on Feldenkrais Method being used among COPD patients. It is therefore the objective of this study to explore the effects of the Feldenkrais Method as compared to the usual pulmonary rehabilitation program (PRP) on Borg score and distance walking. The findings can provide the opportunity for the application of concepts of Feldenkrias Method to be used to complement the existing pulmonary rehabilitation programs and thus benefiting COPD patients.

\section{Methods}

\section{Study Design}

This study is a continuity of a previous study carried out among COPD patients who had undergone 8 weeks of pulmonary rehabilitation program (PRP) with a control group that no intervention. Only 66 subjects completed attendance to the PRP in phase 1 of the study, and they were then enrolled into the study for the second phase of study, which may see patients enrolled into the PRP program (PG) or to take part in the Feldenkrais Program (FG). However, only 17 (47\%) subjects from Feldenkrais group (FG) and another 19 (53\%) from the pulmonary group (PG) managed to complete their intervention. Reasons for withdrawal from the study trial includes 5 $(17 \%)$ subjects that developed exacerbations of COPD whilst the other 25 (83\%) of them voluntary withdrew from the program due to various personal issues such as transportation availability, lack of assistance, failure to get off from work and financial issues.

In phase 1 of the study, recruitment of COPD patients was performed by medical doctors were referred by the physiotherapy department, at University Kebangsaan Malaysia Medical Centre (UKMMC). This was a quasiexperimental study using convenience sampling among a pool of COPD patients who met the inclusion criteria. The study protocol was approved by the medical research and ethics committee of the institution. Written informed consent was obtained prior to enrollment. Eligible patients that met the inclusion criteria included: (1) patients diagnosed as COAD by respiratory physician regardless of their status of disease severity as classified by ATS (2004), (2) able to understand Bahasa Malaysia or English and (3) of aged more than 45 years. Exclusion criteria are patients with malignancy and other severe cardiopulmonary and neurological dysfunctions.

\section{Multidimensional Assessment}

In phase 1 of the study, multidimensional assessment was done at week 1 and 8 and in phase 2 , assessment was done at week 10 and 16 .

\section{Pulmonary Function Testing, 6 minute walk test and Borg test}

Spirometry was performed prior and after each rehabilitation session using spirometry COSMED Pony Spirometer Graphic. Values of forced expiratory volume in 1 second (FEV1) were recorded. Patients were asked to repeat the procedure 3 times and the best reading was taken. The 6-Minute Walk Test (6MWT) is an index of functional capacity with subjects walking as far as they could in 6 minutes. The test was performed along a $30 \mathrm{~m}$ corridor and the better of 2 repeatable tests were taken and the distance walked was recorded. (8) They were encouraged during the test by the therapist every minute. $A$ mean improvement of $54 \mathrm{~m}$ was taken as the cut off point to represent the minimal distance improved among COPD patients (9). With the Borg scale, it allows the patients to grade the intensity of their breathlessness during the walking tests (10). This tool was used as a guide to increase the exercise tolerance among COPD patients. 
The intervention carried out was within 45 to 60 minutes, twice per week and for 8 weeks duration. Patients were recruited randomly into the FG or PG programs.

\section{The Feldenkrais Program (FG)}

The Feldenkrais Method was based upon the work of Moshe Feldenkrais (11). An "Awareness Through Movement" (ATM) lesson was carried out on COAD patients' with exercise regimens different from the usual PRP, prior to starting the exercises. The focus of the program is the restoration of mechanical properties of the human motion by application of sensory motor control of movement. Some of the foundational principles underlying Feldenkrais concepts include paying attention to the quality of movement, doing the movements slowly with minimal effort, resting frequently between movements to avoid physical and mental fatigue, and avoiding pain and discomfort. This activities included relaxation exercises, breathing exercises, floor activities that included sessions of trunk and pelvic movements (rolling on the floor from side to side, trunk twisting, pelvis rolling forward and backward, sitting cross legged).

\section{Pulmonary Rehabilitation Program (PG)}

The exercise sessions in PRP consist of exercise training using bicycle ergometer, lower and upper-extremity training. These patients used an electronically braked bicycle ergometer and they started training at $50 \%$ of the maximum workload. The goal of cycle ergometry was to maintain a training duration of 20 minutes and only then was the work cycled increased according to the individual needs of each patient. Upper-extremity training was performed by repetitively raising and lowering a dowel from the height of the waist to the height of the shoulders (using an interval-training regimen with repetitive period of exercise and rest as tolerated by the patient for 10 minute, a weight of $0.51 \mathrm{~b}$ was added to each arm (12).Treadmill was incorporated for another 15 minutes sessions as tolerated by the patient. Stretching of the limbs muscles are performed prior to each exercise session, for warming up.

\section{Statistical analysis}

After checking for normal distribution, baseline data from the above outcome measures were analyzed using ANOVA analysis which is a two-way mixed split plot design. A significance level was set at $p<0.05$. The magnitude of the effect size was judged against the criteria recommended by Cohen to represent small, moderate and large changes (14).

\section{Results}

There were uneven numbers of males and females subjects between recruited between groups (Table 1). The mean age is younger $(63.53 \pm 9.87)$ in the $F G$ group as compared to PG group (67.52 \pm 9.65$)$. History of smoking was presents in both groups, with more smokers than non-smokers among the subjects. According to classification of disease severity (ATS, 2004), most subjects had moderate degree of airflow obstruction (42\%, $n=15)$, some had mild airflow obstruction $(30 \%, n=11)$ while others had severed airflow obstruction ( $28 \%, n=10)$. The demographic profiles were comparable $(p>0.05)$ for gender $(p=0.048)$, age $(p=0.459)$, and smoking history $(p=0.535)$.

Table 1: $\quad$ Patient characteristic between Feldenkrais and Pulmonary Rehabilitation Group.

\begin{tabular}{|c|c|c|c|}
\hline & $\begin{array}{c}\text { Feldenkrais } \\
\text { Group (IG) n } \\
(\%)\end{array}$ & $\begin{array}{c}\text { Pulmonary } \\
\text { Group (CG) } \\
\text { n (\%) }\end{array}$ & $\begin{array}{c}p \\
\text { value }\end{array}$ \\
\hline \multicolumn{4}{|l|}{ Gender } \\
\hline Male & $7(41.2)$ & $14(73.7)$ & 0.048 \\
\hline Female & $10(58.8)$ & $5(26.3)$ & \\
\hline Age & $63.53 \pm 9.87$ & $67.52 \pm 9.65$ & 0.459 \\
\hline \multicolumn{4}{|l|}{ Race } \\
\hline Malay & $7(41.2)$ & $11(57.9)$ & 0.587 \\
\hline Chinese & $8(47.1)$ & $6(31.6)$ & \\
\hline Indian & $2(11.8)$ & $2(10.5)$ & \\
\hline \multicolumn{4}{|l|}{ Smoking History } \\
\hline Non smokers & $8(47.1)$ & $7(36.8)$ & 0.535 \\
\hline Smokers & $9(52.9)$ & $12(63.2)$ & \\
\hline \multicolumn{4}{|c|}{ Classification of Disease Severity in COPD (FEV\% predicted) } \\
\hline $\begin{array}{l}<30 \% \text { ( Severe } \\
\text { airflow) }\end{array}$ & $4(23.5)$ & $6(31.6)$ & 0.799 \\
\hline $\begin{array}{l}\text { 31-50 (Moderate } \\
\text { airflow) }\end{array}$ & $8(47.1)$ & $7(36.8)$ & \\
\hline 51-80 (Mild airflow) & $5(29.4)$ & $6(31.6)$ & \\
\hline FEV1 in \% predicted & $40.50 \pm 9.72$ & $39.44 \pm 13.38$ & 0.789 \\
\hline Borg Score & $6.00 \pm 0.94$ & $5.84 \pm 1.08$ & 0.632 \\
\hline 6 Minute Walk Test & $315.29 \pm 117.16$ & $323.68 \pm 76.54$ & 0.799 \\
\hline
\end{tabular}

Independent t-test, Significant at ${ }^{*} p<0.05 ;{ }^{* *} p<0.01$

\section{Evaluation of Lung Function Testing}

The FEV1\% predicted showed significant differences within-subjects effect, $F(1,34)=0.447, p<0.001$, partial eta squared $=0.553$, in the Feldenkrias groups (FG). The pulmonary group showed similar status of FEV1\% predicted, $F(1,34)=, p p=0.587$ partial eta squared $=0.021$, across the time periods ( Table 2).

\section{Evaluation of Borg Score}

In the Borg score, there are significant difference in the CG within the groups effect $F(1,34)=0.191, p<0.001$, partial eta squared $=0.809$ indicating better lung volume following intervention compared to baseline reading (Table 
2). The IG proved to benefit more during the intervention with decreased dyspnea. However, the Feldenkrais group had similar Borg score as in the baseline. These showed no improved perception of dyspnea in the FG. Borg score was assessed when subjects were carrying out the 6 minute walked distance (6MWD).

Table 2: $\quad$ Pre and Post values of Borg scale, 6 minute walked distance and FEV1\% predicted following intervention

\begin{tabular}{lllllll}
\hline & \multicolumn{2}{c}{ Pre exercise } & \multicolumn{2}{c}{ Post exercise } & \multicolumn{2}{c}{$p$ value } \\
\hline & IG & CG & IG & CG & $\begin{array}{l}\text { Within } \\
\text { groups } \\
\text { effect }\end{array}$ & $\begin{array}{l}\text { Between } \\
\text { groups } \\
\text { effect }\end{array}$ \\
\hline Borg scale & $6.06+$ & $5.84+$ & $6.00+$ & $3.58+$ & $<0.000^{*}$ & 0.987 \\
(unit) & 1.09 & 1.01 & 0.94 & 1.17 & & \\
6MWT & $302.64 \pm$ & $277.58 \pm$ & $356.58 \pm$ & $282.93 \pm$ & $<0.001 *$ & 0.876 \\
(m) & 107.04 & 79.01 & 93.552 & 72.97 & & \\
FEV1\% & $40.50+$ & $39.44+$ & $42.79+$ & $39.93+$ & 0.403 & 0.626 \\
preicted & 9.72 & 13.38 & 13.10 & 14.66 & & \\
\hline
\end{tabular}

IG= Feldenkais Group, CG- pulmonary rehabilitation group, $p<$ 0.05

\section{Evaluation of 6 Minute Walk Distance (6MWD)}

The exercise tolerance in COPD patients as measured by 6MWD showed significant differences within-subjects effect, $F(1,34)=0.447, p<0.001$, partial eta squared $=$ 0.553 , with both groups showing an increase in 6 Minute Walk Distance (6MWD) across the time periods. The IG proved to benefit more during the intervention with increased distance walked (score differences of $42.46 \mathrm{~m}$ ) compared to CG (score differences of $33.33 \mathrm{~m}$ ). Sessions of Feldenkrais program had enhanced the exercise tolerance of COPD patients much more than the usual PRP. Interestingly it was observed in the FG group, 9 (53 $\%)$ subjects had increased more than 54 meters distance walk compared to only $8(42 \%)$ subjects who had decreases tolerance of less than $54 \mathrm{~m}$. Unlike the PG, only 3(16\%) subjects had an increase of more than 54 meters distance walked (Figure 1). More subjects in the FG group were walking further than 54 meters. An improvement in walking distance of $20 \%$ was observed in FG but only $15 \%$ in the PG.

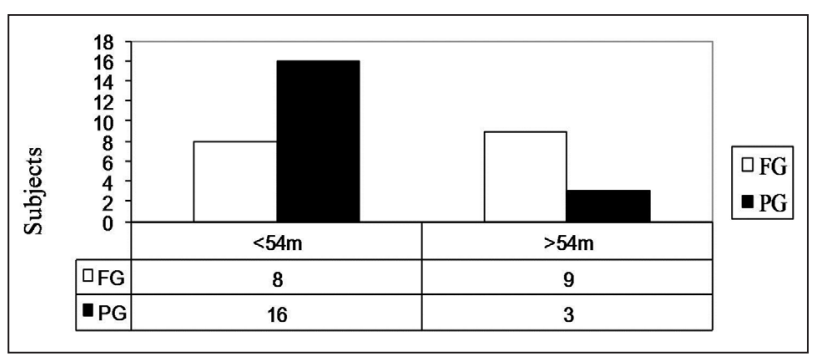

Figure 1: Increase in 6 Minute Distanced Walked Among COPD undergoing Feldenkrais Method and Pulmonary Rehabilitation Program

\section{Discussion}

The COPD patients in this study have extended their rehabilitation for 8 weeks duration. Therefore, it is not surprising that $50 \%$ of them eventually withdrew from the intervention programs since the duration of the study was long and thus not well tolerated. The withdrawal from the program was disappointing but the difficulty in retaining patients in the outpatient programs is well recognized (14). Similar to other studies, the frequency of exacerbations in COPD is difficult to sustained motivation among COPD patients made worse with the severity of the disease (15). The adherence to pulmonary rehabilitation is a major problem as that being reported previously (16) .

The results of the lung function demonstrate only a low improvement in the exercise tolerance of COPD patients. Measures of FEV1 alone are not sufficient to define the treatment response, as this marker does not fully reflect the pervasive nature and burden associated with COPD. Even though there were changes in the mean scores, this was not large enough to be considered significant. Similarly, the differences between groups, in FEV1 (\% predicted) were insignificant to cause any change in the lung function. Therefore, other measures can be used to determine their response to intervention.

Measurements of dyspnoea, for example can provide an insight into the practical effects of treatment on everyday life. This would reflect whether or not the patients perceive an improvement in their primary symptom of COPD since it is a primary reason for them to seek medical care. The significant change in the mean Borg scores have a major influence on the subject. Borg scale have been used to rate perceived dyspnea and exertion. It seems to provides a reliable measure of targeting the training intensity carried out among $\operatorname{COPD}(17,18)$. A subjective rating of 4 to 6 on the Borg scale was appropriate to determine the perception of dyspnoea (19). Those in the PG were observed to be less dyspnea (reduced Borg score) at the later assessment than those in the FG who had almost similar mean score as the initial assessment.

The training in pulmonary rehabilitation involves high intensity exercise and increased cardiovascular endurance. The alteration in the perception of dyspnea in the PG could be an effect of structured physical training. Unlike the FG, the mode of exercise carried out was slow, repetitious and demanded the patients to relax. The exercises were interspersed with breathing naturally and at their own pace. The similar Borg score observed in the initial assessment during the walking tests truly represents an exercise mode-specific desensitization. Throughout the sessions, the physiotherapist was guiding the subjects through a sequence of gentle non-strenuous movements. Attentiveness achieved among the subjects helps them to discover how to move more comfortably and efficiently. There were comments made by the subjects of their experiences with immediate improvements in posture, a feeling of lightness of movement, and freedom from chronic discomforts. The only limitation in this study, 
however that is no measurement of minute ventilation (VE) and oxygen consumption (VO2 maximum) was carried out during the course of the intervention.

There were improvements reported in the breathing patterns of subjects undergoing Feldenkrais program. Subjects perceived themselves as always having breathlessness and there is fear of losing control of themselves and in their minds, their Borg score was always similar to what was identified earlier in the initial assessment. The individual's perception of dyspnea is subjected to the subjects' familiarization of what they perceived as being dyspnoea. In using the Borg score, it is important to note that the scoring requires knowledge or quantification of the stimulus among the subjects. Poor subject understanding of how the scores are measured, can be possible reasons for the poor scores perceived by the subjects. This may be difficult to establish because of lack of clear differences between scores. This is consistent with the study of Knox and colleagues (1988), who reported improvements in visual analog scores for dyspnoea over three days of repeated testing but not over four weeks suggesting that the reduced dyspnea was part of a learning effect or related to the changing attitude and beliefs which are known to be among the strong predictors of exercise performance (20).

The 6 minute walking distance is generally believed to be representative of activities of daily living such as shopping, as the level of exertion associated with this test is considered to be maximal (21). Such modest increases in walking distance could therefore be translated into clinically meaningful improvements in quality of life especially if it is accompanied by improvement in symptoms. The improved walking distance in FG is consistent with true physiological training effect. Since the Feldenkrais sessions did not have any structured physical exercise, the improved walking performance in this group presumably occurred because of the improved ventilator capacity which requires adaptations for it to significantly improve the exercise performance (22).

The application of various movement pattern carried out during Feldenkrais sessions indirectly causes a manual stretch of a single or several intercostals spaces which is said to be a therapeutic procedure to improve gas exchange. These techniques have shown to improve regional ventilation, gas exchange, respiratory muscle function, dyspnea, exercise tolerance, and quality of life (23). Compared to spontaneous breathing, pursed-lip breathing reduces respiratory rate, dyspnea, and arterial pressure of carbon dioxide ( $\mathrm{PaCO} 2$ ), while improving tidal volume and oxygen saturation in resting conditions (24).

The findings is consistent with an early meta analysis that supports the view of a beneficial effect of relaxation on dyspnoea (25). This was later confirmed by a pilot study that evaluated the role of breathing and relaxation training in patients with severe COPD (26), where patients were randomized either to the intervention or a control (standard care) group. Although, symptoms scored on SGRQ and the MRC Dyspnoea Score appeared unchanged over the test period, there was a significant differences in the walking distance (six minute walking test) between the two groups. The improvements in walking distance occurred without any increase sensation of dyspnoea suggesting the beneficial effect of relaxation in the management of exercise induced dyspnea, which was consistent with our findings.

The implication of our study provides opportunity for a different approach of management to be carried out among COPD patients. This is especially beneficial for severed COPD who tolerates less intense exercise as in PRP but able to progress slowly with Feldenkrais Method. Unfortunately, this study is with limitation as there are reasonably large number of patients who had dropped out of the study recruitment, hence resulting in small number of subjects in the second phase of study. A possible cross over effect of the pulmonary rehabilitation program in Phase 1 could have had an influencing effect on the improvement in Phase 2 of the study. However, further research is warranted to identify possible predictors of improvement with Feldenkrais Method.

\section{Conclusion}

Both intervention demonstrated relevance to COPD patients however; the PRP remains the best method to increase patient's tolerance in exercise. Whilst it may be the case that COPD patients may better tolerate the Feldenkrais program, it does not provide better outcomes. Further studies regarding this may be necessary to justify its use in clinical practice since the present study only recruited a small number of subjects.

\section{Acknowledgement}

The author wishes to thank the medical officer who helps with patient recruitment at the respiratory clinic and the staff of physiotherapy department for making this study a success.

\section{References}

1. Ferrer M, Alonso J, Morera Jea. Chronic obstructive pulmonary disease stage and health-related quality of life. The Quality of Life of Chronic Obstructive Pulmonary Disease Study Group. Ann Intern Med 1997; 127: 072-079.

2. Jenkins SC, Cecins NM, Collins GB. Outcomes and direct costs of a pulmonary rehabilitation service. Physiother Theory Pract. 2001;17:67-76.

3. Pauwels RA, Buist AS, Calverley PM, Jenkins CR, Hurd SS. Global strategy for the diagnosis, management, and prevention of chronic obstructive pulmonary disease. NHLBI/WHO Global Initiative for Chronic Obstructive Lung Disease (GOLD)Workshop summary. Am J Respir Crit Care Med 2001;163:1256-76. 
4. Hopper C, Kolt GS, McConville JC. The effects of Feldenkrais Awareness Through Movement on hamstring length, flexibility, and perceived exertion. Journal of Bodywork and Movement Therapies. 1999;3:238-47.

5. Kearney J. The Feldenkrais Method in the secondary school., A case study report. Unpublished masters thesis, La Trobe University Melbourne. 1994.

6. Mahler DA, Tomlinson D, Olmstead EA, Tosteson ANA, O'Connor GT. Changes in dyspnoea, health status and lung function in COAD. Am J Respir Crit Care Med. 1995;151:61-5.

7. Lacasse $\mathrm{Y}$, Brosseau L, Milne S, et al. Pulmonary rehabilitation for chronic obstructive pulmonary disease. Cochrane Database of Systematic Reviews (4) Article CD0003793. 2006 [10 September 2006].

8. American Thoracic Society. ATS statement: Guidelines for the six-minute walk test. Am J Respir Crit Care Med. 2002;166:111-7.

9. Redelmeier DA, Bayoumi AM, Goldstein RS, Guyatt GH. Interpreting small differences in functional status: the six minute walk test in chronic lung disease patients. Am J Respir Crit Care Med 1997;155:1278-82.

10. Borg GA. Psychophysical bases of perceived exertion. Med Sci Sports 1982;14:377-81.

11. Feldenkrais M. Awareness Through movement. New York, NY: Harper Collins. 1972.

12. Celli RB. Exercise training in pulmonary rehabilitation. Semin Respir Med 1993. 1993;14:132-8.

13. Cohen J. Statistical Power Analysis For The Behavioural Sciences. New York, Academic Press. 1978.

14. Sewel IL, Singh SJ, Williams JE, Collier R, Morgan MD. How long should outpatient pulmonary rehabilitation be? A randomized controlled trial of 4 weeks versus 7 weeks. Thorax. 2006;61(9):767-71.

15. Burge PS, Calverley PM, Jones PW, Spencer S, Anderson JA, Maslen TK. Randomised, double blind, placebo controlled study of fluticasone propionate in patients with moderate to severe chronic obstructive pulmonary disease: the ISOLDE trial. BMJ 2000;320:1297-303.
16. Arnold MEH, Hill EC, Gibson D, Bruton A. What factors influence adherence to pulmonary rehabilitation in patients with chronic obstructive pulmonary disease? . Australian Journal of Physiotherapy 2006;52(1):57.

17. Mejia R, Ward J, Lentine T, Mahler DA. Target dyspnea ratings predict expected oxygen consumption as well as target heart rate values. Am J Respir Crit Care Med. 1999; 159:1485-9.

18. Horowitz MB, Littenberg B, Mahler DA. Dyspnea ratings for prescribing exercise intensity in patients with COPD. Chest 1996;109:1169-75.

19. Puente-Maestu L, Sanz ML, Sanz P, Cubillo JM, Mayol J, Casaburi R. Comparison of effects of supervised versus self-monitored training programmes in patients with chronic obstructive pulmonary disease. Eur Respir J 2000;15:517-25.

20. Knox AJ, Morrison JF, Muers MF. Reproducibility of walking test results in chronic obstructive airways disease. Thorax. 1988;43:388-92.

21. Kervio G, Carre F, Ville NS. Reliability and intensity of the six.minute walk test in healthy elderly subjects. Med Sci Sports Exerc ;35:169-74. 2003;35:169-74.

22. Mador MJ, Deniz O, Aggarwal A, Shaffer TJ, Kufel J, Spengler CM. Effect of respiratory muscle endurance training in patients with COPD undergoing pulmonary rehabilitation. Chest. 2005; 128:1216-24.

23. Gosselink R. Controlled breathing and dyspnea in patients with chronic obstructive pulmonary disease. J Rehabil Res Dev 2003;40:25-34.

24. Bianchi R, Gigliotti F, Romagnoli I, et al. Chest wall kinematics and breathlessness during pursedlip breathing in patients with COPD. Chest 2004;125:45965.

25. Devine EC, Pearcy J. Meta-analysis of the effects of psycho educational care in adults with chronic obstructive pulmonary disease. Patient Education and Counselling 1996;29:167-78.

26. Eiser N, West C, Evans S, Jeffers A, Quirk F. Effects of psychotherapy in moderately severe COPD: a pilot study. European Respiratory Journal 1997;10:1581-4. 